\title{
Insights into the metabolomes of parasites
}

\begin{abstract}
'Metabolomics', the study of the profile of metabolites present in a cell/organism, has matured to the extent that it is beginning to provide exciting information and insights into how cells/organisms are adapted to their environments. The technology is now more widely available and so applying it to studies on parasites is feasible. Thus the British Society for Parasitology (http://www.bsp.uk.net/) held a meeting in September 2009 at the University of Strathclyde, UK entitled 'Insights into the Metabolomes of Parasites' to give an update of what can be done and how, the power that this approach possesses, and the possibilities of applying the methods to address key research questions in parasitology. The assembled speakers covered all aspects of metabolomics from sample preparation, through data acquisition, to data analysis and interpretation. This compilation of reviews arose from the
\end{abstract}

presentations at the meeting, although each is more comprehensive in content and aims to provide all of the material required for those contemplating undertaking metabolomic studies. It is to be hoped that this Special Issue of Parasitology will be the springboard for many more metabolomic investigations on parasites in the next decade, studies which will provide seminal new insights into the metabolism of parasites and so lay the foundations for the new antiparasite therapies of the future.

Graham H. Coombs Strathclyde Institute of Pharmacy and Biomedical Sciences University of Strathclyde

Glasgow United Kingdom

April 2010 